\title{
Lactoferrin Suppresses LPS-Induced Expression of HMGB1, microRNA 155, 146, and TLR4/MyD88/NF- KB Pathway in RAW264.7 Cells
}

\section{Maryam Nemati}

Islamic Azad University

\section{Saeideh Akseh}

Tabriz University of Medical Sciences

\section{Maryam Amiri}

Jiroft University of Medical Sciences

\section{Hamid Reza Nejabati}

Tabriz University of Medical Sciences

\section{Ahmadreza Jodati}

Tabriz University of Medical Sciences

\section{Nazila Fathi Maroufi}

Tabriz University of Medical Sciences

Yousef Faridvand ( $\nabla$ y.faridvand@gmail.com )

Tabriz University of Medical Sciences https://orcid.org/0000-0003-1139-3500

\section{Mohammad Nouri}

Tabriz University of Medical Sciences

\section{Research}

Keywords: Lactoferrin, TLR4-MyD88-NF-kB, HMGB1, MicroRNA, RAW264.7

Posted Date: October 23rd, 2020

DOl: https://doi.org/10.21203/rs.3.rs-40992/v2

License: (c) (1) This work is licensed under a Creative Commons Attribution 4.0 International License. Read Full License

Version of Record: A version of this preprint was published at Immunopharmacology and Immunotoxicology on January 12th, 2021. See the published version at https://doi.org/10.1080/08923973.2021.1872616. 


\section{Abstract}

Objective: This current study evaluated the underlying mechanisms of LF against the inflammatory microRNAs (miRNAs), HMGB1 expression and TLR4-MyD88-NF-KB pathway in LPS-activated murine RAW264.7 cells.

Methods: MTT assay was used to assess cell metabolism and the cell culture levels of the cytokines (TNF-a, IL-6) were evaluated by Enzyme-linked immunosorbent assay (ELISA). The expression of miRNAs was quantified by using qPCR and expression of HMGB1, TLR4, MyD88, and phosphorylated NF-KB (Pp65) were determined with Western blot and qPCR, respectively.

Results: The results indicated that LF downregulates IL-6 and TNF- $a$ expression. LF exhibited the degradation of P-p65 and reduced the production of HMGB1, TLR4, and MyD88 in LPS-induced inflammatory response. Importantly, in parallel with the suppression of cytokines and HMGB1-TLR4MyD88-NF-KB pathway, LF could induce a decrease in inflammatory selected miRNAs, mmu-mir-155 and mmu-mir-146a expression.

Conclusions: Altogether, these findings provide LF as a prominent anti-inflammatory agent could modulate HMGB1, mmu-mir-155, mmu-mir-146a and TLR4/MyD88/NF-KB pathway.

\section{Introduction}

Inflammation causes activating and releasing of pro-inflammatory cytokines in response to infections via complex networks of interaction. The cytokines then trigger an appropriate inflammatory response and play a pivotal role in removing the infection [1]. However, abundant and poorly regulated cytokines expression in responses to the inflammation has been confirmed to be highly involved with cell and tissue injury in inflammatory-associated disease, including, atherosclerosis, sepsis, and infections [2]. Lipopolysaccharide (LPS) is a Gram-negative bacteria product, which has been commonly used to induce inflammatory response by activation of macrophage Toll-like receptor 4 (TLR4)[3].

Toll-like receptor4 (TLR4) is a conserved mediator of inflammation, up-regulate the expression of its downstream effectors, most importantly NF-KB, through MyD88 adaptor [4]. High mobility group box-1 (HMGB1), a damage-associated molecule pattern (DAMP) protein, an early mediator of inflammation, is released in passive form from impaired cells and activates the innate immune cell. In addition, it has been found that macrophages cells actively secrete HMGB1, which enhances inflammatory reaction [5]. HMGB1 also binds to TLR4 receptor and promotes the NF-KB activity through TLR4/MyD88/NF-KB dependent pathway, thereby; ablation of HMGB1 could be protective against the further production of cytokines and cell damage [6]. Moreover, HMGB1 has previously been shown to act as a potent inducer of proinflammatory cytokines production, which amplifies their activity in acute inflammation [7]. Therefore, targeting HMGB1 may provide an important therapeutic strategy against inflammatory response through TLR4-mediated signaling. 
MicroRNAs (miRNAs), one class of small non-coding RNA (sncRNA), modulate gene regulation. Evidence reveals that miRNAs are potent regulators of macrophage activation and polarization [8]. Studies on miRNAs expression profile noticed that some miRNAs expression such as, mmu-mir-223, mmu-mir-146a and $m m u-m i r-155$ are involved in NF-kB levels in activated macrophage $[9,10]$. Recent evidence has reported that LPS-mediated activation of TLR-signaling pathways by induction of mmu-mir-146a and mmu-mir-155 expression [11-13]. Moreover, mmu-mir-146a and mmu-mir-155 expression in response to LPS stimulation has been blocked by inhibition of NF-kB activity in Raw264.7 cells [14,15]. Liu et al demonstrated that mmu-mir-129 inhibited the development of AE-related epilepsy by negatively targeting HMGB1 protein and abrogated TLR4/NF-kB signaling activation by HMGB1[16].

It has been suggested that lactoferrin (LF), one of the major antioxidants in human milk, has potential pharmacological activities, could reduce tissue inflammatory response in T cells [17]. Our previous findings and other reports have suggested that LF inhibit the inflammatory response to the high-fat dietinduced inflammation and metabolic stress in rat $[18,19]$. It was also informed that LF significantly attenuates the catecholamine-induced cardiotoxicity by inhibition of reactive oxygen species (ROS) [20]. Studies have shown that LF inhibited the production of tumor necrosis factor- $a$ and nitric oxide-induced by LPS [21]. A recent study reported that LF reduces the inflammatory response by inhibition of TLR4SIRT-1-NFKB signaling cascade in obese diabetic pediatric population [22]. By our knowledge, no previous experimental study has been done to investigate the effects of LF on the inflammation-related signaling pathway, TLR4-MyD88-NF-KB, and HMGB1. Therefore, this current study was conducted to examine the effect of LF on the TLR4-MyD88-NF-KB pathway and also provided evidence of LF effects on selected miRNAs expression in LPS-activated Raw264.7 cells.

\section{Materials And Methods}

\section{Reagents}

Fetal bovine serum (FBS), Dulbecco's modified Eagle's medium (DMEM), and penicillin-streptomycin were purchased from Gibco (Carlsbad, CA, USA). LF was from Sigma-Aldrich (St. Louis, MO, USA).

Lipopolysaccharide (LPS) (Escherichia coli 0127, B8) was purchased from Sigma.

\section{Cell culture}

Macrophage RAW 264.7 cells were purchased (Pasteur Institute, Tehran) and cultured in DMEM medium, supplemented with $10 \% \mathrm{FBS}, 1 \%$ of penicillin/streptomycin at $37^{\circ} \mathrm{C}$ in $5 \% \mathrm{CO} 2$ incubator. Cells at $80 \%$ confluence were subcultured and the passages $3-5$ were subjected to the subsequent experiment.

\section{Cell metabolism and morphology}

Cell metabolism was measured by MTT assay. Briefly, RAW264.7 cells were grown into 96-well plates $\left(10^{4}\right.$ cells/well) for an overnight. Then, cell treatment was done with $\operatorname{LF}(0,10,100,500$ and $1000 \mu \mathrm{g} / \mathrm{mL})$ and incubated for $1 \mathrm{~h}$, and then were treated with LPS $(1 \mu \mathrm{g} / \mathrm{mL})$. After $24 \mathrm{~h}$, MTT solution $(0.5 \mathrm{mg} / \mathrm{mL})$ 
was added and incubated for further $4 \mathrm{~h}$. Then, after dissolving of formazan crystals by DMSO, the optical density was measured at $570 \mathrm{~nm}$ (Bio-Tek, ELX 800, USA). The qualitative morphology of the cell cultures was also analyzed by a light microscope.

\section{Detection of miRNA and mRNA expression}

Total RNA with miRNA contents were isolated from the RAW264.7 cells by using TRIzol reagent (SigmaAldrich, Germany). Extracted RNAs quality was assessed using Nanodrop (Thermo Scientific). The cDNA synthesis was conducted with the cDNA synthesis kit (Takara, Shiga, Japan) by using Total RNA $(1 \mu \mathrm{g})$. The stem-loop-based quantitative real-time RT-PCR (qRT-PCR) method was used for detecting miRNAs expression. The qRT-PCR analyses were performed using Takara SYBR ${ }^{\circledR}$ Premix Ex Taq (Takara, Japan) on a LightCycler ${ }^{\circledR} 96$ System (Roche Diagnostics, Germany). miRNAs primers were designed according to the corresponding sequence from miRBase and NCBI database and were listed in Table 1 . The $2^{-\triangle \triangle C T}$ method was applied for calculation of the relative expressions of the genes.

\section{Assessment of TNF- $a$ and IL- 6 expression}

Cells were seeded in 24-well plates $\left(10^{6}\right.$ cells $\left./ \mathrm{mL}\right)$ for $24 \mathrm{~h}$, and then were treated with LF and stimulated with LPS for indicated periods. The concentration of TNF-a and IL-6 in cell culture of LF with or without LPS groups was carried out the commercially available ELISA kit (ExCell Biotech, China).

\section{Western blot}

For western blotting, cells were collected and washed using ice-cold PBS. Thereafter, cells were homogenized by lysis buffer (Roche Diagnostics, Germany). Protein concentration was quantified using a NanoDrop (ND-1000 Spectrophotometer, USA). Extracted protein was separated by $12 \%$ SDS-PAGE and blotted to a PVDF membrane (Millipore, USA), then, blocked and hybridized with the specific antibodies (Santa Cruz, CA, USA). After incubation with secondary antibodies, the results were visualized by enhanced chemiluminescence (Thermo Fisher Scientific, USA) in the dark-room. The results were normalized to the GAPDH signal value in the same lane.

\section{Data analysis}

Results are expressed as the mean $\pm S D(n=3)$. The one-way analysis of variance (ANOVA) with multiple comparisons was used to compare the differences between the groups using GraphPad Prism software (Version 6; GraphPad, CA). A $p<0.05$ was considered to as significant

\section{Results}

\section{Effect of LF on cell morphology and cell viability}

LPS induces morphological transformation of macrophage RAW264.7 cells. As shown in Fig. 1, LPS induced activation and morphological changes on RAW264.7 cells, while this transformation was 
decreased by LF pretreatment in concentrations of 500 and $1000 \mu \mathrm{g} / \mathrm{ml}$ of LF. Further quantitative analysis of cell metabolism was detected using the MTT assay. As shown in figure 1, with LPS challenge, cell metabolism was found to be decreased and this reduction was significant relative to control cells $(p<0.05)$. Pretreatment of RAW264.7 with LF restored cell metabolism resulted from LPS treatment and $1000 \mu \mathrm{g} / \mathrm{ml}$ of LF had the maximal protective effect in comparison with LPS treatment alone $(\mathrm{p}<0.05)$. Therefore, in our subsequent in vitro experiments, LF was used in RAW 264.7 cells at the concentration of 500 and $1000 \mu \mathrm{g} / \mathrm{mL}$ for $24 \mathrm{~h}$ in further experiments.

\section{LF inhibits LPS-induced IL-6 and TNF- $a$ expression}

To explore the potent anti-inflammatory property of LF, we determined the production of LPS-induced IL-6 and TNF-a expression in RAW264.7 cells. As data showed, LPS significantly increased IL-6 and TNF-a in macrophage cells. Interestingly, pretreatment with LF decreased LPS-induced IL- 6 and TNF-a expression at mRNA level $(p<0.05)$; (Fig. 2A). Results demonstrated that LF also notably decreased the protein levels of IL- 6 and TNF- $a$ as compared with the LPS group ( $p<0.05$ ); (Fig. 2B). However, these results indicated that LF reduced the pro-inflammatory cytokine production in a concentration-dependent manner.

\section{LF regulates miRNAs expression in LPS-induced RAW264.7 cells}

We next explored whether LF could inhibit the transcription on the regulation of mmu-mir-155 and $m m u^{-}$ mir-146a. As shown in Fig. 3, the expression levels of the miR-155 and miR-146a were altered by LPS treatment in RAW264.7 cells. RAW264.7 cells were pretreated with LF and then stimulated with LPS for 24 h. Both $m m u-m i r-155$ and mmu-mir-146a were rapidly induced in response to LPS and significantly reduced when pretreated with LF (Fig.3). Therefore, LF inhibited the expression of both inflammatoryassociated miRNAs; suggesting that LF may act upstream to inhibit the transcription of the mmu-mir-155 and mmu-mir-146a genes.

\section{LF suppress TLR4/Myd88/NF-KB pathway}

As shown in Fig.4, TLR4/Myd88/NF-KB pathway has a major role in the activation of the inflammatory cascades. The results indicated that LPS-induced significant up-regulation in HMGB1, TLR4, and Myd88 genes. However, LF significantly attenuated HMGB1, TLR4, and Myd88 proteins levels in RAW264.7 cells and more reduction was observed at the concentrations of $1000 \mu \mathrm{g} / \mathrm{ml}$ of LF compared with $500 \mu \mathrm{g} / \mathrm{ml}$. The results demonstrated that the levels of HMGB1, TLR4, and Myd88 were blocked in the LPS treatment group (Fig. 4A, 4B, and 4C). We additionally evaluated the phosphorylation of P65 (p-P65), a functional subunit of NF-KB, a master regulator of inflammation, and found highly expressed levels of P-p65 in LPSinduced cells. However, P-p65 levels were found to be significantly declined in concentrations of 500 and $1000 \mu \mathrm{g} / \mathrm{ml}$ of LF. LF had no significant effect on the total protein level of p-P65, HMGB1, TLR4, and Myd88 protein levels in the control group.

\section{Discussion}


LF administration has been provided as a dietary component for the treatment of inflammation disorder, including arthritis, allergy, and cancer [23]. Antioxidant and anti-inflammatory activity of LF has already been proved by numerous experimental studies $[24,25]$. Because of the beneficial effects of LF, experimental studies paid more interest to investigate the underlying mechanism action of LF [26]. Excessive release of cytokines may result in pathological changes by inducing wide and reverse tissue damage [27]. Studies indicated that the ablation of TLR4 by either knockout or TLR4 antagonist decreases NF-KB levels in LPS activated macrophage, suggesting that inhibition of TLR4 might be pivotal to impede chronic inflammation [28]. Our study suggests new insights into the regulation of HMGB1 and TLR4-MyD88-NF-KB pathway by LF in LPS-activated RAW264.7 cells. We observed that LF exerted antiinflammatory against LPS-induced cytokine production in RAW264.7 cells. In our study, the expression levels of HMGB1 (ligand for TLR4) was increased parallel with the increase of TLR4 as well as the MyD88 and NF-KB in the LPS challenge. However, LF significantly mitigated all these pathological changes by a reduction in TLR-4, MyD88, NF-KB and HMGB1 levels in LPS-activated cells. However, these results provided that LF pretreatment inhibits HMGB1 production and its downstream TLR4-MyD88-NFKB pathway. Therefore, it is highly likely that LF may prevent, at least partially, if not fully, the inflammatory response through targeting the HMGB1 and TLR4-MyD88-NF-KB pathway. In addition, in the present study, LPS increased pro-inflammatory cytokines IL-6 and TNF- $\alpha$ and this adverse effect was inhibited by Lf intervention. A similar beneficial effect of LF was observed on other models; Inubushi et al. reported that Lf impeded LPS-induced IkBa and IKK $\beta$ phosphorylations, the two important related kinases for DNA binding activity of NF-KB [29]. Wei et al showed that LF extended the survival time of radiated mice and improved intestinal injury by decreasing TNF-a, IL-6, and NF-KB [30]. Na et al confirmed that pretreatment LF-LPS complex induces LPS tolerance in RAW 264.7 cells by a reduction in IL-1 $\beta$, IL-6, and TNF-a production. They also revealed the modulation of NF-KB DNA binding activity by LF-LPS treatment [31]. Mohamed et al, also demonstrated that LF promotes activation of PPAR- $\gamma$ and SIRT-1 and inhibited TLR4 downstream NF-KB signaling pathway in type 2 diabetic [22].

The LPS-induced miRNAs regulation in RAW264.7 cells has been revealed the immune-regulatory effects of miRNAs under inflammatory response [32]. However, the protective effect of LF against inflammation by regulation of miRNAs is another major challenge that remains to be clear. In addition, it has been reported that LPS-induced aberrant expression of miRNAs in RAW264.7 cells, and associated with an amplified inflammatory response [33]. Our findings showed that LPS increased $m m u-m i r-155$ and $m m u-$ mir-146a expression, which could be significantly reduced under LF treatment. We further showed that LF pretreatment inhibited mmu-mir-155 and mmu-mir-146a expression at the concentrations of 500 and $1000 \mu \mathrm{g} / \mathrm{ml}$ of LF. To the best of the authors' knowledge, the current study is the first to investigate the effects of LF on mmu-mir-155 and mmu-mir-146a expression in LPS activated RAW264.7 cells. MiRNAs are strongly linked to immune responses and cytokines regulations; among the miRNAs, mmu-mir-155 and mmu-mir-146a have been found to promote the inflammation. To date, mmu-mir-155 and mmu-mir146 induction have been involved in aberrant expression of genes associated with innate immune response [34,35]. mmu-mir-155 expression is strongly induced by LPS in macrophages and positively regulates the inflammatory axis upon pathogenic activation of TLR4 [36]. Further studies have shown 
that the decreased mmu-mir-155 contributes to inhibit TLR-4 induced IL- 6 and IFN- $\beta$ expression in macrophages [15]. Additionally, the expression of mmu-mir-146a has been found to be upregulated in RAW264.7 macrophage cells in response to LPS stimulation [35] and down-regulation of LPS-mediated mmu-mir-155 and mmu-mir-146a expressions play an important role by TLR4/NF-KB pathway activity [14]. Therefore, the inhibition of mmu-mir-155 and mmu-mir-146a has been associated with a decrease in TLR-4 mediated NF-KB activity [37]. Consistent with previous studies [38], in this current study, inhibition of P-p65 and TLR4 by LF elicited a downregulation of mmu-mir-155 in LPS treated cells, which indicates the important role of the NF-KB pathway in LF-regulated $m m u-m i r-155$ expression. In addition, as we indicated above, LF attenuated the expression of HMGB1, TLR4, MyD88 and NF-KB (p-P65) by LPS stimulation; suggesting the suppression of HMGB1 and TLR4-MyD88-NF-KB pathway may be associated with the effect of LF on expression of mmu-mir-146a and mmu-mir-155 in response to LPS stimulation. This study provides plausible evidence that LF induces significant changes in mmu-mir-146a and $m m u-$ mir-155 expression particularly in LPS activated inflammation in RAW264.7 cells. Although the human and mouse immune system exhibit extensive similarities [39] but identify differences in human and mouse macrophages in response to LF administration under LPS stimulation warrants further exploration and it could be limitation of the study. However, additional mechanistic investigations are required to explore possible targeted genes and pathways affected by the LF.

In summary, we demonstrate that LF exhibited anti-inflammatory effects by attenuating TNF- $a$, IL- 6 , and HMGB1 levels through the TLR4-MyD88-NF-KB pathway and miRNAs expression in LPS-activated macrophage cells. We also provided the first evidence on the effects of LF on the regulation of selected miRNAs expression but further in vivo studies are warranted to explore the relationship between miRNAs and inflammatory genes.

\section{Declarations}

\section{Acknowledgments}

We thank the members of our laboratory for their technical help

\section{Author contribution}

Yousef Faridvand and Mohammad Nouri conceived and designed the research; Maryam Nemati, Mohammad-Ali Karimi, Maryam Amiri, Hamid Reza Nejabati, Nazila Fathi Maroufi, and Yousef Faridvand performed the experiments; Yousef Faridvand analyzed the data; Hamid Reza Nejabati drafted the text and wrote the paper.

\section{Funding}


This work was supported by the Student Research Committee, Tabriz University of Medical Sciences, Tabriz, Iran

\section{Availability of data and materials}

The data that support the findings of this study are available from the corresponding author upon request

\section{Ethics approval and consent to participate}

The experimental protocol was approved by the Ethics Committee of Tabriz Medical University

\section{Consent for publication}

Not applicable.

\section{Competing interests}

The authors declare no conflicts of interest.

\section{References}

1. Nathan C. Points of control in inflammation. Nature. 2002;420: 846-852.

2. Levi M, van der Poll T, Schultz M. Infection and inflammation as risk factors for thrombosis and atherosclerosis. Semin Thromb Hemost. 2012;38: 506-514.

3. Han XZ, Ma R, Chen Q et al. Anti-inflammatory action of Athyrium multidentatum extract suppresses the LPS-induced TLR4 signaling pathway. J Ethnopharmacol. 2018;217:220-227.

4. Chithra MA, Ijinu TP, Kharkwal $\mathrm{H}$ et al. Phenolic rich Cocos nucifera inflorescence extract ameliorates inflammatory responses in LPS-stimulated RAW264.7 macrophages and toxin-induced murine models. Inflammopharmacology. 2019.

5. Lee DU, Ko YS, Kim HJ et al. 13-Ethylberberine reduces HMGB1 release through AMPK activation in LPS-activated RAW264.7 cells and protects endotoxemic mice from organ damage. Biomed Pharmacother. 2017;86: 48-56.

6. Elfeky M, Yoneshiro T, Okamatsu-Ogura Y et al. Adiponectin suppression of late inflammatory mediator, HMGB1-induced cytokine expression in RAW264 macrophage cells. J Biochem. 2018;163: 143-153.

7. Hreggvidsdottir HS, Ostberg T, Wahamaa $\mathrm{H}$ et al. The alarmin HMGB1 acts in synergy with endogenous and exogenous danger signals to promote inflammation. J Leukoc Biol. 2009;86: 655662. 
8. Essandoh K, Li Y, Huo J et al. MiRNA-Mediated Macrophage Polarization and its Potential Role in the Regulation of Inflammatory Response. Shock. 2016;46: 122-131.

9. M'Baya-Moutoula E, Louvet L, Molinié R et al. A multi-omics analysis of the regulatory changes induced by miR-223 in a monocyte/macrophage cell line. Biochimica Et Biophysica Acta (BBA)Molecular Basis of Disease. 2018;1864: 2664-2678.

10. Nahid MA, Satoh M, Chan EK. MicroRNA in TLR signaling and endotoxin tolerance. Cellular \& molecular immunology. 2011;8: 388-403.

11. Cobos Jiménez V, Bradley EJ, Willemsen AM et al. Next-generation sequencing of microRNAs uncovers expression signatures in polarized macrophages. Physiological genomics. 2014;46: 91103.

12. Lu D, Yamawaki T, Zhou H et al. Limited differential expression of miRNAs and other small RNAs in LPS-stimulated human monocytes. PloS one. 2019;14: e0214296.

13. Taganov KD, Boldin MP, Chang K-J et al. NF-KB-dependent induction of microRNA miR-146, an inhibitor targeted to signaling proteins of innate immune responses. Proceedings of the National Academy of Sciences. 2006;103: 12481-12486.

14. Jin S, Wang J, Chen $S$ et al. A novel limonin derivate modulates inflammatory response by suppressing the TLR4/NF-kappaB signalling pathway. Biomed Pharmacother. 2018;100: 501-508.

15. Sun Y, Cai J, Ma F et al. miR-155 mediates suppressive effect of progesterone on TLR3, TLR4triggered immune response. Immunol Lett. 2012;146: 25-30.

16. Liu A-H, Wu Y-T, Wang Y-P. MicroRNA-129-5p inhibits the development of autoimmune encephalomyelitis-related epilepsy by targeting HMGB1 through the TLR4/NF-kB signaling pathway. Brain research bulletin. 2017;132: 139-149.

17. Wolf JS, Li G, Varadhachary A et al. Oral lactoferrin results in T cell-dependent tumor inhibition of head and neck squamous cell carcinoma in vivo. Clin Cancer Res. 2007;13: 1601-1610.

18. Faridvand $Y$, Nozari S, Asoudeh-Fard A et al. Bovine lactoferrin ameliorates antioxidant esterase activity and 8-isoprostane levels in high-cholesterol-diet fed rats. Int J Vitam Nutr Res. 2017;87: 201206.

19. Nozari S, Fathi Maroufi N, Nouri M et al. Decreasing serum homocysteine and hypocholesterolemic effects of Bovine lactoferrin in male rat fed with high-cholesterol diet. J Cardiovasc Thorac Res. 2018;10: 203-208.

20. Mladenka P, Semecky V, Bobrovova Z et al. The effects of lactoferrin in a rat model of catecholamine cardiotoxicity. Biometals. 2009;22: 353-361.

21. Choe YH, Lee SW. Effect of lactoferrin on the production of tumor necrosis factor-alpha and nitric oxide. J Cell Biochem. 1999;76: 30-36.

22. Mohamed WA, Schaalan MF. Antidiabetic efficacy of lactoferrin in type 2 diabetic pediatrics; controlling impact on PPAR-gamma, SIRT-1, and TLR4 downstream signaling pathway. Diabetol Metab Syndr. 2018;10: 89. 
23. Lepanto MS, Rosa L, Paesano R et al. Lactoferrin in Aseptic and Septic Inflammation. Molecules. 2019;24.

24. Mulder AM, Connellan PA, Oliver CJ et al. Bovine lactoferrin supplementation supports immune and antioxidant status in healthy human males. Nutr Res. 2008;28: 583-589.

25. Gonzalez-Chavez SA, Arevalo-Gallegos S, Rascon-Cruz Q. Lactoferrin: structure, function and applications. Int J Antimicrob Agents. 2009;33: 301 e301-308.

26. Tammam SN, Azzazy HME, Lamprecht A. Nuclear and cytoplasmic delivery of lactoferrin in glioma using chitosan nanoparticles: Cellular location dependent-action of lactoferrin. Eur J Pharm Biopharm. 2018;129: 74-79.

27. Lee HJ, Maeng K, Dang HT et al. Anti-inflammatory effect of methyl dehydrojasmonate (J2) is mediated by the NF-kappaB pathway. J Mol Med (Berl). 2011;89: 83-90.

28. Kim HM, Park BS, Kim Jl et al. Crystal structure of the TLR4-MD-2 complex with bound endotoxin antagonist Eritoran. Cell. 2007;130: 906-917.

29. Inubushi T, Kawazoe A, Miyauchi M et al. Molecular mechanisms of the inhibitory effects of bovine lactoferrin on lipopolysaccharide-mediated osteoclastogenesis. J Biol Chem. 2012;287: 2352723536.

30. Wei YL, Xu JY, Zhang R et al. Effects of lactoferrin on X-ray-induced intestinal injury in Balb/C mice. Appl Radiat Isot. 2019;146: 72-77.

31. Na YJ, Han SB, Kang JS et al. Lactoferrin works as a new LPS-binding protein in inflammatory activation of macrophages. International immunopharmacology. 2004;4: 1187-1199.

32. Cheng Y, Kuang W, Hao $Y$ et al. Downregulation of miR-27a* and miR-532-5p and upregulation of miR-146a and miR-155 in LPS-induced RAW264.7 macrophage cells. Inflammation. 2012;35: 13081313.

33. He X, Jing Z, Cheng G. MicroRNAs: new regulators of Toll-like receptor signalling pathways. Biomed Res Int. 2014;2014: 945169.

34. Cheng HS, Njock MS, Khyzha $\mathrm{N}$ et al. Noncoding RNAs regulate NF-kappaB signaling to modulate blood vessel inflammation. Front Genet. 2014;5: 422.

35. He Y, Sun $X$, Huang $C$ et al. MiR-146a regulates IL-6 production in lipopolysaccharide-induced RAW264.7 macrophage cells by inhibiting Notch1. Inflammation. 2014;37: 71-82.

36. Piccinini AM, Midwood KS. Endogenous control of immunity against infection: tenascin-C regulates TLR4-mediated inflammation via microRNA-155. Cell Rep. 2012;2: 914-926.

37. Wu XY, Fan WD, Fang R et al. Regulation of microRNA-155 in endothelial inflammation by targeting nuclear factor (NF)-kappaB P65. J Cell Biochem. 2014;115: 1928-1936.

38. Zheng $Y$, Xiong $S$, Jiang $P$ et al. Glucocorticoids inhibit lipopolysaccharide-mediated inflammatory response by downregulating microRNA-155: a novel anti-inflammation mechanism. Free Radic Biol Med. 2012;52: 1307-1317. 
39. Mestas J, Hughes CC. Of mice and not men: differences between mouse and human immunology. The Journal of Immunology. 2004;172: 2731-2738.

\section{Figures}
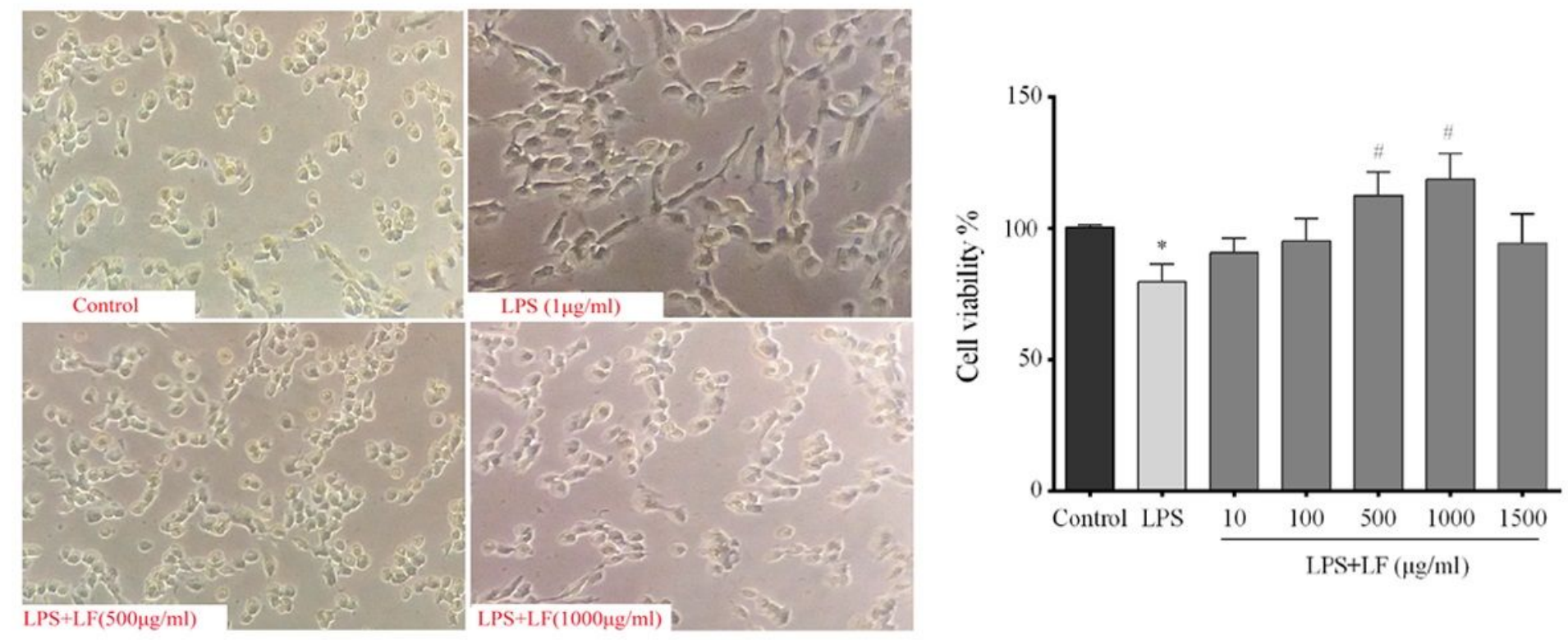

\section{Figure 1}

Effect of LF on the viability of RAW264.7 cells. Cells were incubated with LF $(0,10,100,500$, and 1000 $\mu \mathrm{g} / \mathrm{mL}$ ), and then stimulated with lipopolysaccharide (LPS; $1 \mu \mathrm{g} / \mathrm{ml}$ ) for $24 \mathrm{~h}$. Cell morphology was monitored under a light microscope at $x 400$ magnitude and cell viability was performed by using MTT assay. The data are expressed as means $\pm S D$. ${ }^{\star} p<0.05$ vs. control group; $\# p<0.05$ vs. LPS group $(n=3)$. 
A
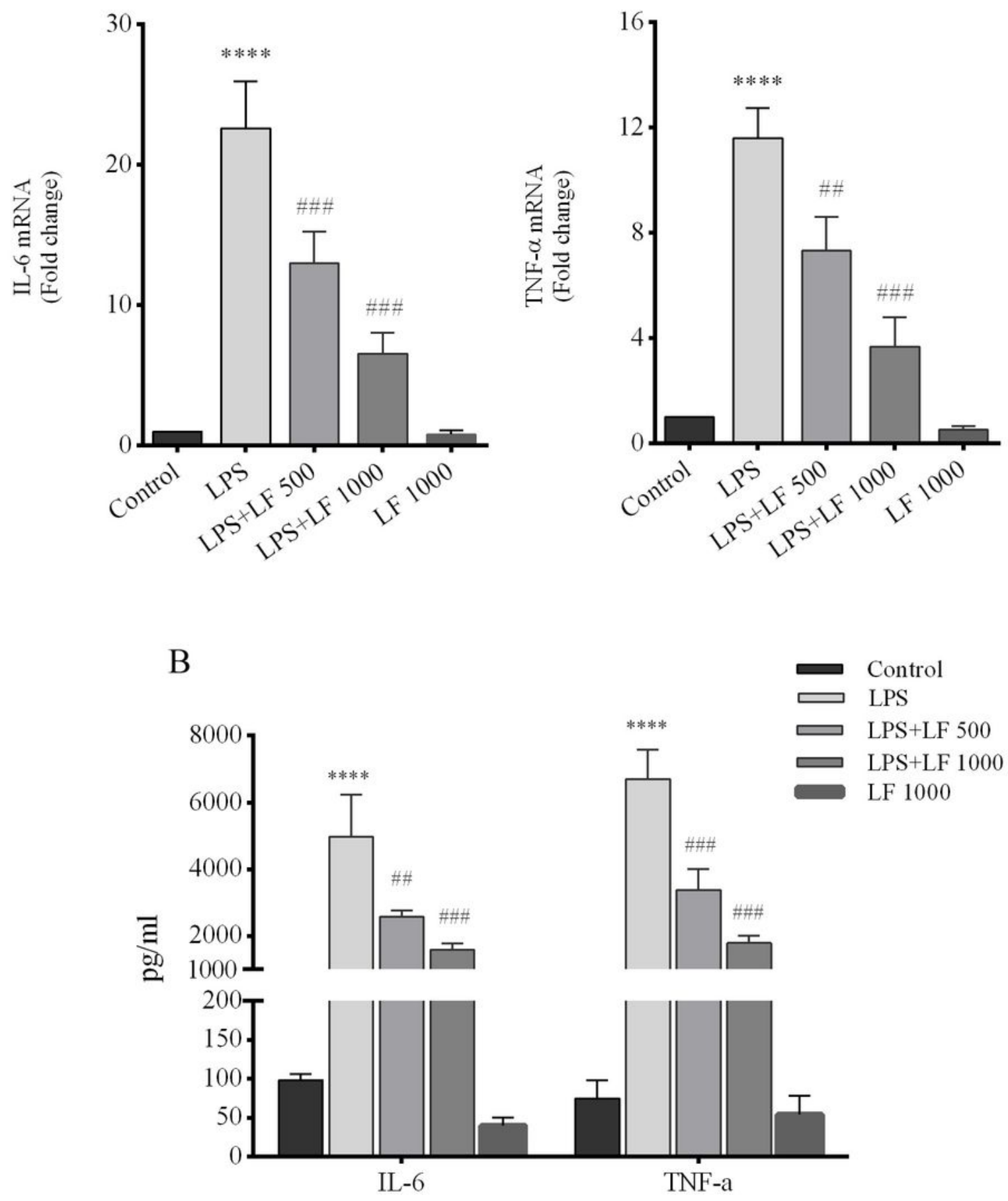

Figure 2

Effect of LF on LPS-induced IL-6 and TNF-a expression in RAW264.7 cells. Cells were pretreated with LF (500, and $1000 \mu \mathrm{g} / \mathrm{mL}$ ) and exposed to $1 \mu \mathrm{g} / \mathrm{ml}$ LPS for $24 \mathrm{~h}$. (A) IL-6 and TNF-a gene expression were detected using real-time qPCR. (B) Protein levels of IL-6 and TNF-a in RAW264.7 cells was detected using an ELISA kit. The data are expressed as means \pm SD. $\star \star \star \star x p<0.0001$ vs. control group; \#\#\#p $<0.001$, and $\# \# \# p 0.001$ vs. LPS group $(n=3)$ 


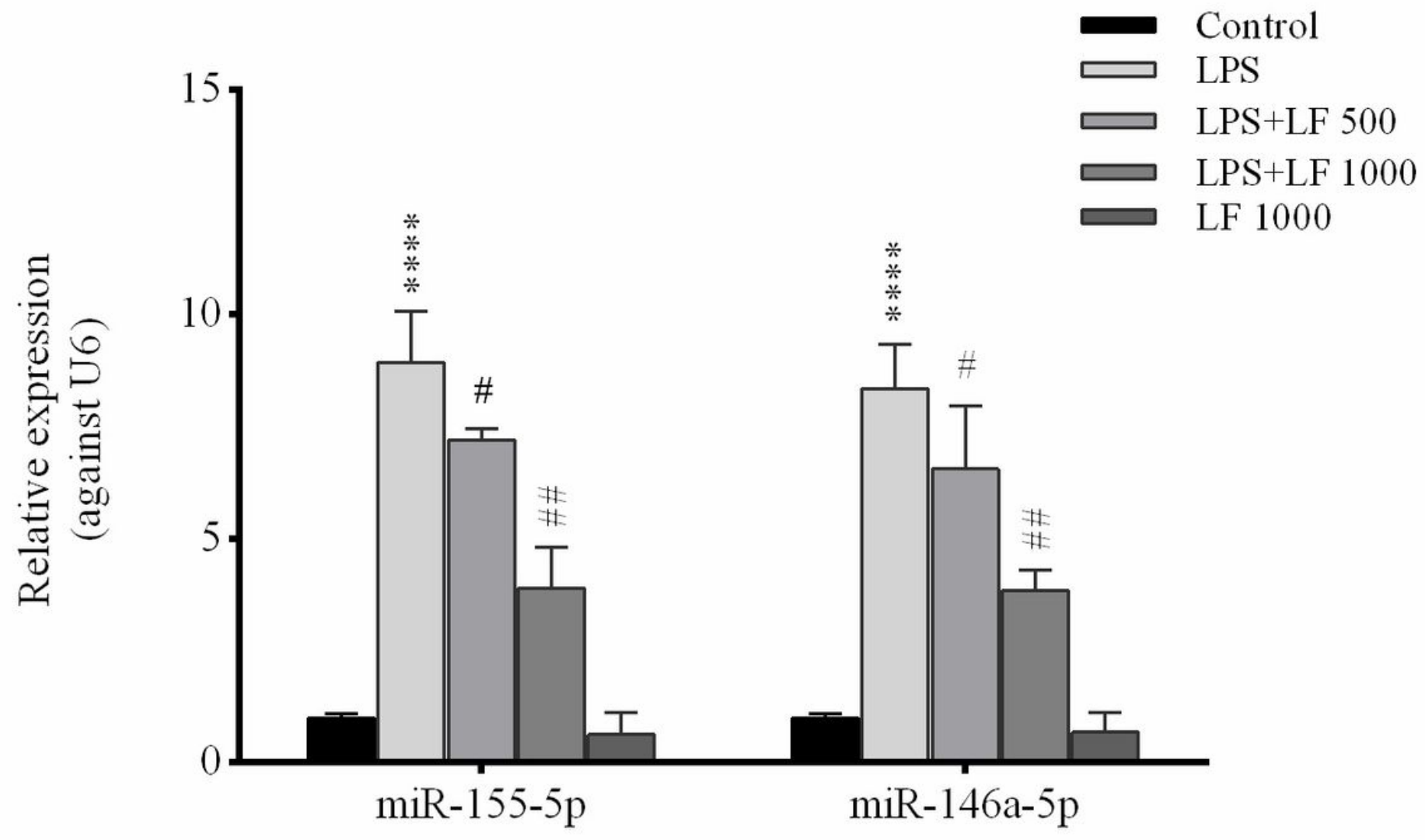

Figure 3

Effect of LF on LPS-induced miRNAs expression in RAW264.7 cells. Cells were pretreated with AME (500, and $1000 \mu \mathrm{g} / \mathrm{mL}$ ) and exposed to $1 \mu \mathrm{g} / \mathrm{ml}$ LPS for $24 \mathrm{~h}$. (A) And (B) The expression of different miRNAs were measured by using real-time $q P C R$. The data are expressed as means $\pm S D$. ${ }^{*} p<0.05,{ }^{\star} p<0.01$, $\star \star \star \star p<0.0001$ vs. control group; $\# p<0.05, \# \# p<0.01$ vs. LPS group $(n=3)$ 
A
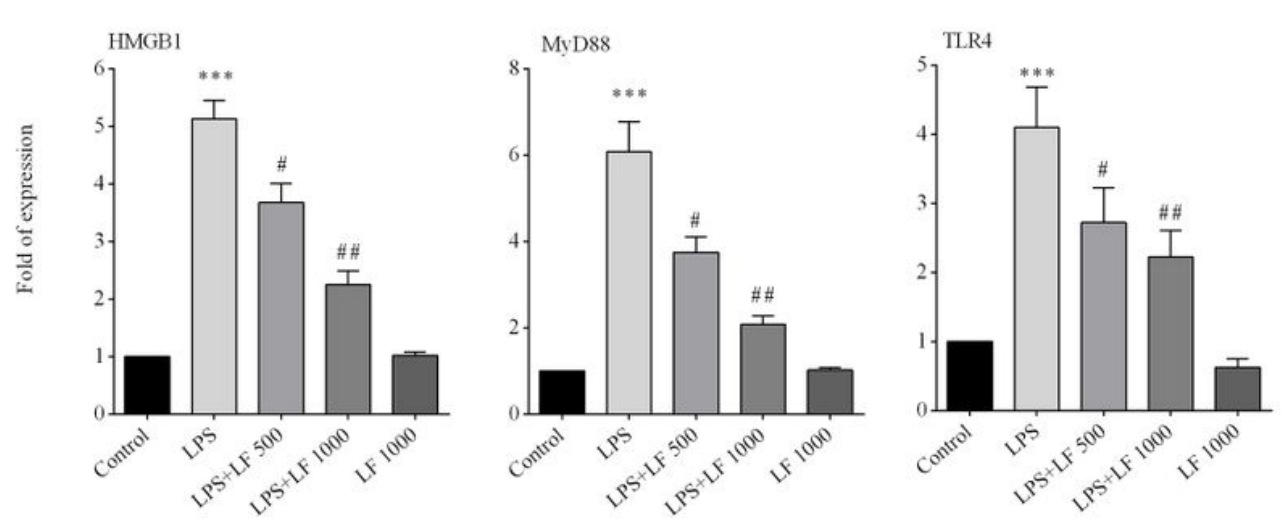

B

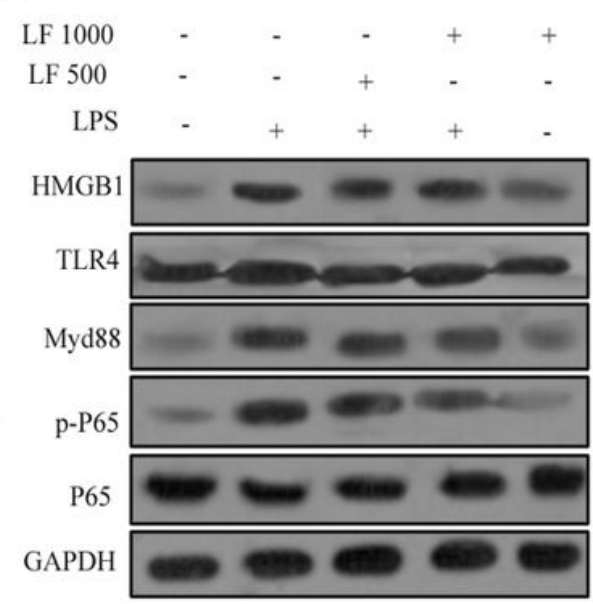

$\mathrm{C}$

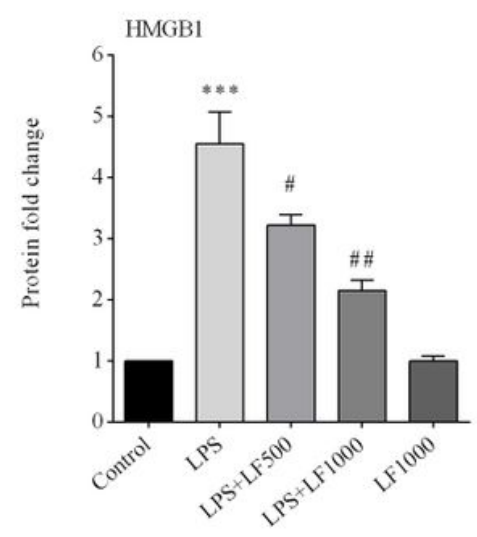

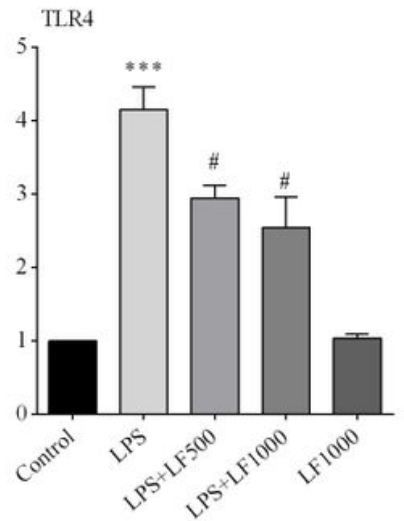

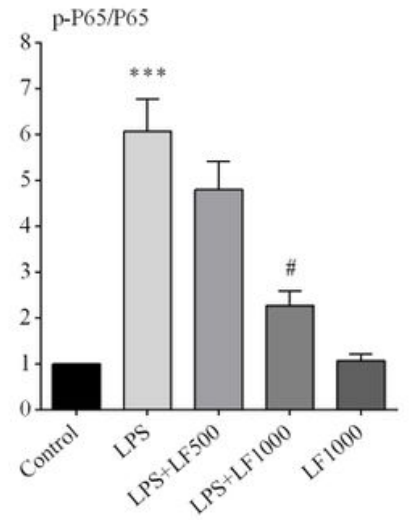

\section{Figure 4}

Effect of LF on LPS-induced HMGB1-TLR4-MyD88-NF-KB pathway expression in RAW264.7 cells. Cells were pretreated with LF $(500$, and $1000 \mu \mathrm{g} / \mathrm{mL})$ and exposed to $1 \mu \mathrm{g} / \mathrm{ml}$ LPS for $24 \mathrm{~h}$. The expression of proteins and mRNA samples were measured using real-time qPCR and western blot, respectively. (A) The relative expression of HMGB1, Myd88, and TLR4 genes. (B) Representative immunoblot image of protein samples. (C) The expression levels of HMGB1, TLR4, Myd88, and NF-KB (P-p65 and p65) were assessed in whole-cell protein extract by Western blot. GAPDH was used as loading control protein. The data are expressed as means \pm SD. ${ }^{*} p<0.05$, ${ }^{\star \star \star} p<0.001$ vs. control group; $\# p<0.05, \# \# p<0.01$ vs. LPS group $(n=3)$ 